\title{
Adiabatic expansion and magnetic fields in AGN jets
}

\author{
Alexander B. Pushkarev*i \\ Max-Planck-Institut für Radioastronomie, Auf dem Hügel 69, 53123 Bonn, Germany \\ Pulkovo Observatory, Pulkovskoe Chaussee 65/1, 196140 St. Petersburg, Russia \\ Crimean Astrophysical Observatory, 98409 Nauchny, Crimea, Ukraine \\ E-mail: apushkar@mpifr-bonn.mpg.de \\ Yuri Y. Kovalev \\ Max-Planck-Institut für Radioastronomie, Auf dem Hügel 69, 53123 Bonn, Germany \\ Astro Space Center of Lebedev Physical Institute, Profsoyuznaya 84/32, 117997 Moscow, Russia \\ E-mail: ykovalev@mpifr-bonn.mpg.de
}

\section{Andrei P. Lobanov}

Max-Planck-Institut für Radioastronomie, Auf dem Hügel 69, 53123 Bonn, Germany

E-mail: alobanov@mpifr-bonn.mpg.de

\begin{abstract}
Results of high-resolution simultaneous multi-frequency 8.1-15.4 GHz VLBA polarimetric observations of relativistic jets in active galactic nuclei (the MOJAVE-2 project) are analyzed. We compare characteristics of VLBI features with jet model predictions and test if adiabatic expansion is a dominating mechanism for the evolution of relativistic shocks in parsec-scale AGN jets. We also discuss magnetic field configuration, both predicted by the model and deduced from electric vector position angle measurements.
\end{abstract}

The 9th European VLBI Network Symposium on The role of VLBI in the Golden Age for Radio Astronomy and EVN Users Meeting

September 23-26, 2008

Bologna, Italy

\footnotetext{
${ }^{*}$ Speaker.

$\dagger$ Acknowledgements. Y.Y. Kovalev is a Research Fellow of the Alexander von Humboldt Foundation. The National Radio Astronomy Observatory is a facility of the National Science Foundation operated under cooperative agreement by Associated Universities, Inc. This research has made use of the NASA/IPAC Extragalactic Database (NED) which is operated by the Jet Propulsion Laboratory, California Institute of Technology, under contract with National Aeronautics and Space Administration. We would like to thank Matt Lister and Tuomas Savolainen for useful comments and discussion.
} 


\section{Introduction}

In spite of the fact that our understanding of active galactic nuclei phenomenon has been significantly improved over the past decade [11], there are many aspects of the nature of the relativistic outflows (jets), like their composition, formation, acceleration, collimation, etc, which remain to be strongly debated. VLBI observations provide us with the unique tool to explore the jets in AGN with milliarcsecond angular resolution corresponding to parsec-scale resolution in linear size. Typically, parsec-scale flows are characterized by one-sided knotty structure with sometimes pronounced curvature, rapid variations of flux density (e.g. [17, 14]), and detection of superluminal motions [3].

Relativistic shocks propagating down the jets are expected to be prominent on these scales, which is confirmed by detection of strong polarization $[12,13]$ and enhancement of the magnetic field in the brightest jet features (from the turnover frequency images) [9]. The general evolution of a shock-induced flare in a jet is considered by [6] to pass through the Compton, synchrotron, and adiabatic stages, referring to the dominant energy loss mechanism. In the current paper we present recent results of testing adiabatic expansion of relativistic shocks in several active galactic nuclei.

Throughout the paper the $\Lambda \mathrm{CDM}$ cosmological model with $H_{0}=70 \mathrm{~km} \mathrm{~s}^{-1} \mathrm{Mpc}^{-1}, \Omega_{m}=0.3$, and $\Omega_{\Lambda}=0.7$ is adopted. Spectral index $\alpha$ is defined as $S \propto v^{\alpha}$.

\section{Observational data in use}

We use experimental data collected and processed in the framework of the MOJAVE-2 ${ }^{1}$ project. The observations were performed by the MOJAVE team in 2006 at VLBA, a VLBI network of ten 25-m telescopes, in a dual-polarization mode, quasi-simultaneously at four frequencies of 8.1, 8.4, $12.1, \& 15.4 \mathrm{GHz}$. The scan lengths were chosen to achieve roughly the same image rms at each observing frequency. The observed sample consists of 192 sources, see for details [8].

\section{Results and Discussion}

Using the shock-in-jet-model [5] we have investigated an evolutionary scenario in which a bright jet feature is treated as an independent, geometrically thin relativistic shock developing in a flow with the emission dominated by adiabatic energy losses. It is also assumed that the jet plasma has a power-law particle energy distribution $N(E) d E \propto E^{-s} d E$. Following [1] and applying Gauss's flux theorem and Lorentz transformations to the cross section of the jet, the magnetic field can be approximated as $B \propto d^{-n}$, where $d$ is the transverse jet size and $n$ describes the orientation of the magnetic field ( $n=1$ for the transverse field and $n=2$ for the longitudinal field). Assuming non-changing Doppler factor along the jet, the model brightness temperature of each jet component, $T_{\mathrm{b} \text {, jet }}$, can be related, as it was shown in [10], to the measured brightness temperature of the core, $T_{\mathrm{b}, \text { core }}$, as $T_{\mathrm{b}, \text { jet }}=T_{\mathrm{b} \text {, core }}\left(d_{\text {jet }} / d_{\text {core }}\right)^{-\xi}$, where $d$ represents the measured sizes of the core and jet components, and $\xi=n+1-\alpha(n+4 / 3)$.

${ }^{1}$ Monitoring Of Jets in Active galactic nuclei with VLBA Experiments, see
http://www.physics.purdue.edu/MOJAVE/


We compare the measured brightness temperatures with those predicted by the model, determining the spectral index of each jet component as well as the B-field orientation (if polarization emission is detected, preferably at $15.4 \mathrm{GHz}$, by rotating the electric vectors by $90^{\circ}$ due to the optically thin synchrotron radiation detected in the jets).

In this paper we discuss the results for two sources, 1128-047 and 2155-152, having prominent outflows with sufficient number of jet components suitable for the analysis.

\section{$3.11128-047$}

This flat-spectrum radio galaxy with redshift $z=0.266(3.91 \mathrm{pc} / \mathrm{mas})$ and mass of the central object of $5.2 \times 10^{6} \mathrm{M}_{\odot}$ [18] shows one-sided jet structure (Fig. 1, left) which was fitted by seven distinct Gaussian components at $8.1 \mathrm{GHz}$. The model fitting was performed in DIFMAP [15]. In case of applying an elliptical Gaussian component for the VLBI core the minor axis of the ellipse has been used to estimate $d$, since the VLBI core component has a strong tendency to be elongated in the innermost jet direction [4].

Using the VLBI images at 8.1 and $12.1 \mathrm{GHz}$, we constructed the spectral index distribution and its slice along the jet ridge line (Fig. 1, right). Since the linear polarization in this source has not been detected down to about $1 \mathrm{mJy}$ level, we calculated the model brightness temperatures for all of the jet components assuming (i) parallel (Fig. 2, left) and (ii) perpendicular magnetic filed configuration (Fig. 2, right). In the latter case, the values of $T_{\mathrm{b}}^{\text {obs }}$ and $T_{\mathrm{b}}^{\text {mod }}$ agree within the errors. This allows us to suggest a predominantly transverse orientation of magnetic field in the jet.
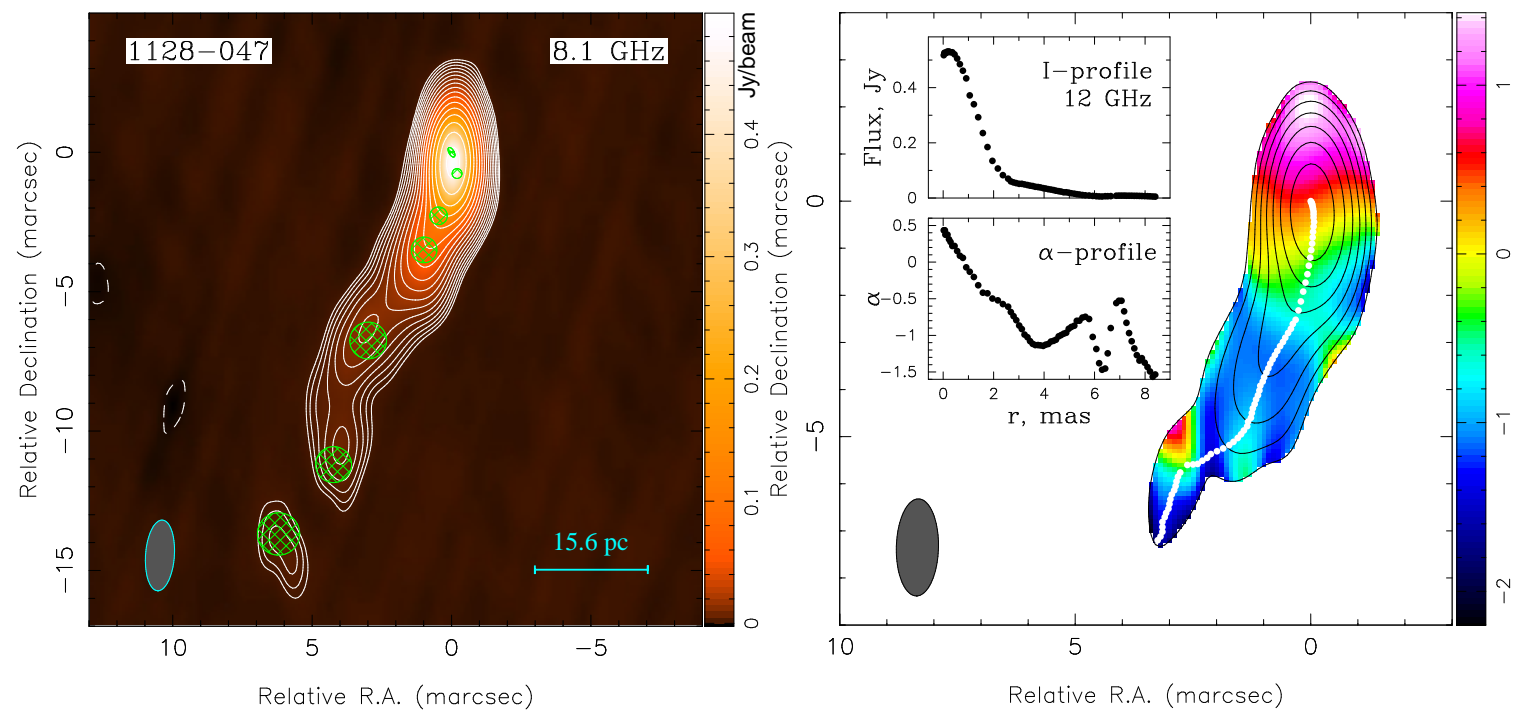

Figure 1: Left panel: Total intensity image of radio galaxy $1128-047$ at $8.1 \mathrm{GHz}$ at epoch of 2006.92 with the model of 7 Gaussian components superimposed. The lowest contour is plotted at $0.35 \%$ of the peak brightness of $501 \mathrm{mJy} /$ beam. Shaded ellipse represents the FWHM of the restoring beams of $2.54 \times$ 1.04 mas at $\mathrm{PA}=-4.0^{\circ}$. Right panel: Spectral index distribution in 1128-047 calculated between 8.1 and 12.1 GHz with the $12.1 \mathrm{GHz}$ total intensity contours overlayed. White dots represent the total intensity ridge line along which we plot the profiles of total intensity and spectral index as an inset image. The lowest contour is plotted at $0.84 \%$ of the peak brightness of $531 \mathrm{mJy} /$ beam. Shaded ellipse represents the FWHM of the restoring beam of $2.06 \times 0.89$ mas at $\mathrm{PA}=-1.4^{\circ}$. 

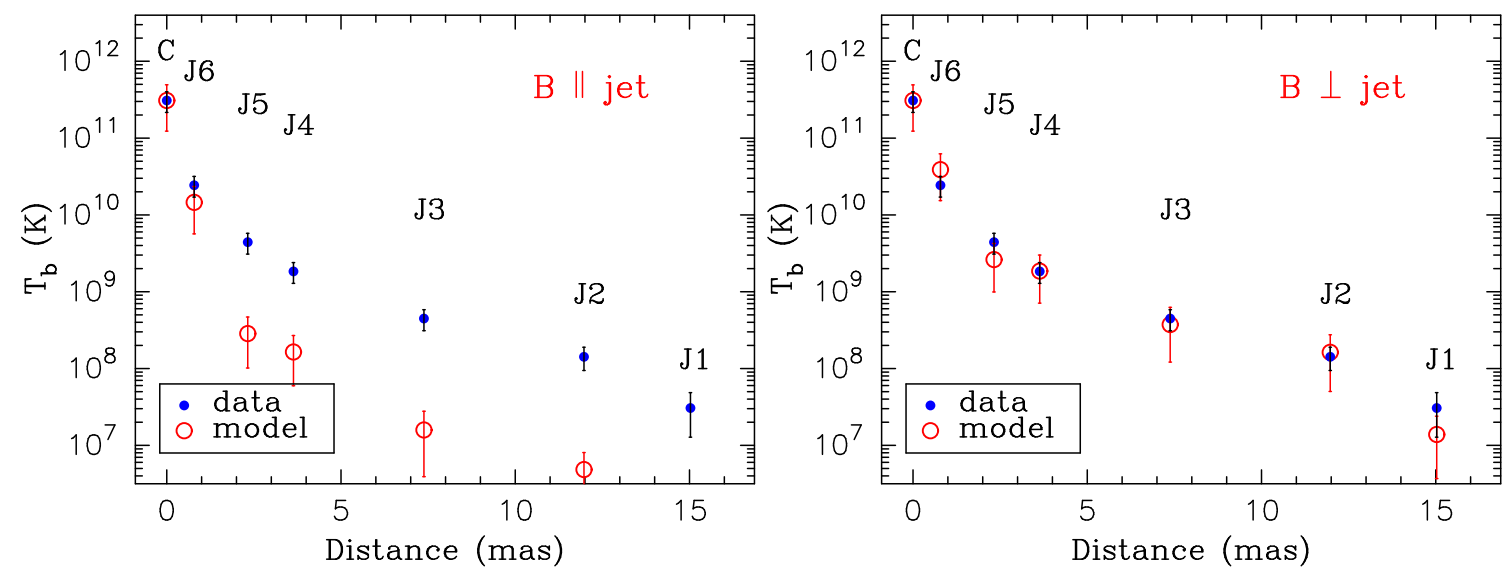

Figure 2: Left panel: Brightness temperature changes along the jet in $1128-047$ at $8.1 \mathrm{GHz}$. Filled blue circles are the measured values. Open red circles are the predicted brightness temperatures from the shockin-jet model with adiabatic losses dominating the radio emission assuming a predominantly longitudinal magnetic field orientation to the local jet direction. The errors are given at $3 \sigma$ level. Right panel: The same data as on the left but assuming a transverse magnetic field orientation. Big discrepancies (left) and remarkable agreement (right) between measured and model brightness temperature values lead to the conclusion of a perpendicular orientation of the magnetic field in the jet.

\section{$3.22155-152$}

This flat-spectrum source with redshift $z=0.672(7.03 \mathrm{pc} / \mathrm{mas})$ and mass of the central object of $3.9 \times 10^{7} \mathrm{M}_{\odot}$ [18] was classified as a BL Lac object by Craine et al. [2] and then as a quasar by Véron-Cetty \& Véron [16] on the basis of its optical spectrum.

Using the parsec-scale spectral index information (Fig. 3, right) taken from the I-maps at 8.1 and $15.4 \mathrm{GHz}$ (Fig. 3, left) together with the results from model fitting, and also assuming a magnetic field perpendicular to the jet, we have calculated the values of $T_{\mathrm{b}}^{\text {mod }}$ and $T_{\mathrm{b}}^{\mathrm{obs}}$. The brightness temperatures predicted by the model agree well with those from the data for all the components (Fig. 4, right), except the optically thin $\mathrm{J} 2$ feature $\left(\alpha_{\mathrm{J} 2}=-0.75\right)$ located at 3.36 mas from the core, for which $\zeta=T_{\mathrm{b}}^{\mathrm{obs}} / T_{\mathrm{b}}^{\bmod } \approx 20$. There are two possible ways to explain this discrepancy:

(i) The $\mathrm{J} 2$ component is the region of the outflow where the particles are reaccelerated through the interaction with the surrounding medium. An evidence for this is the formation of a shear layer as is described in the decelerating jet model [7]: in the central channel, where the magnetic field is predominantly tranverse, the plasma moves with relativistic speed but slows down at the edges due to the interaction with an ambient medium making a sheath of longitudinal magnetic field registered on polarization images (Fig. 4, left).

(ii) The Doppler factor varies in the $\mathrm{J} 2$ region due to changes of the speed or orientation of the jet. The apparent speed $\beta_{\text {app J5 }}=(10.8 \pm 1.3) c$ is determined from the MOJAVE kinematics $[3,8]$. The $\mathrm{J} 2$ component is quasi-stationary (during a period of $5.2 \mathrm{yr}$ the centroid position was wiggling within 0.3 mas in non-radial direction). Then assuming the viewing angle $\theta_{\max } \approx 5.3^{\circ}(\theta \sim 1 / \beta)$ to be constant and applying the analytical adiabatic approximation [1] for a relativistic jet with predominantly transverse B-field, we determined the change of speed $\Delta \beta \leq 0.002$ between the J5 and $\mathrm{J} 2$ regions. Therefore, the detected excess of the Doppler factor $\delta_{\mathrm{J} 2} / \delta_{J 5}=\zeta^{1 /\left(3-\alpha_{\mathrm{J} 2}\right)}=2.22 \mathrm{can}$ be explained by changing the viewing angle that becomes less than the jet opening angle $\phi \approx 1^{\circ}$. 

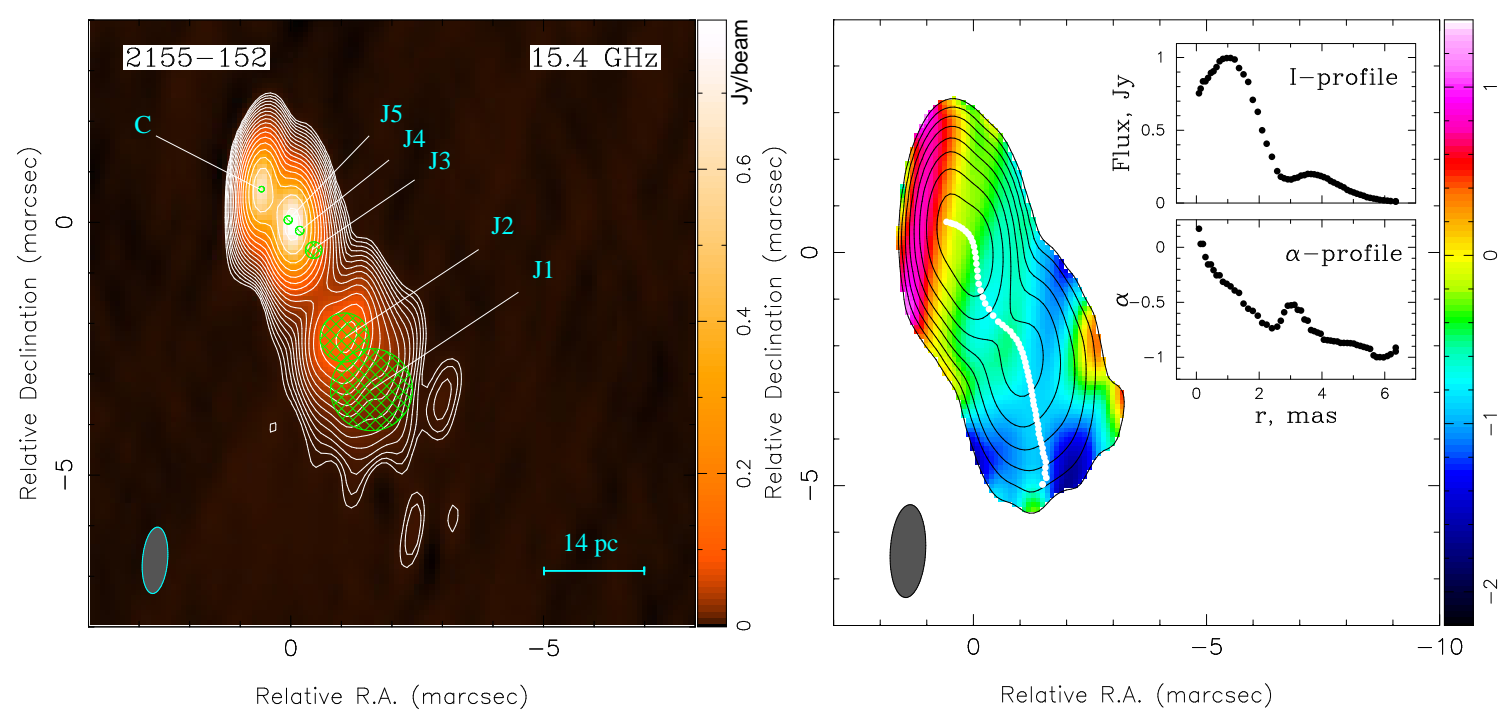

Figure 3: Left panel: Total intensity image of quasar $2155-152$ at $15.4 \mathrm{GHz}$ at epoch 2006.92 with model fitted Gaussian components superimposed. The lowest contour is plotted at $0.24 \%$ of the peak brightness of $796 \mathrm{mJy} /$ beam. Shaded ellipse represents the FWHM of the restoring beam of $1.32 \times 0.50$ mas at PA = $-5.0^{\circ}$. Right panel: Spectral index distribution in $2155-152$ calculated between 8.1 and $15.4 \mathrm{GHz}$ with the 15.4 GHz total intensity contours overlayed. White dots represent the total intensity ridge line along which we plot the profiles of total intensity and spectral index as an inset image. The lowest contour is plotted at $0.45 \%$ of the peak brightness of $1000 \mathrm{mJy} /$ beam. Shaded ellipse represents the FWHM of the restoring beam of $1.99 \times 0.76$ mas at $\mathrm{PA}=-3.2^{\circ}$.
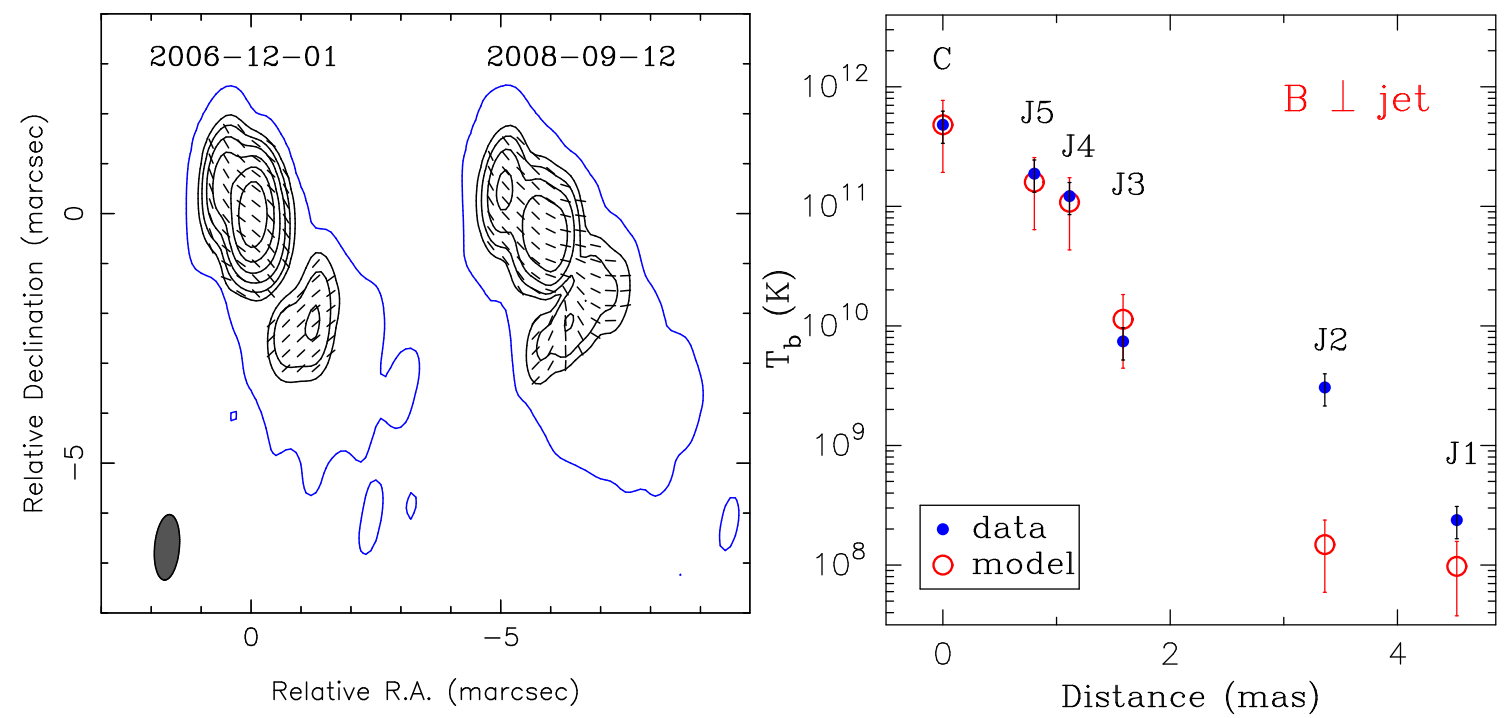

Figure 4: Left panel: Linear polarization images of 2155-152 at $15.4 \mathrm{GHz}$ with electric vectors superimposed on epochs 2006.92 and 2008.70. The bottom P-contour is plotted at $1.8 \mathrm{mJy}$ for both epochs. The blue contour represents the total intensity bottom contour at a level of $1.9 \mathrm{mJy}$ for both epochs. The sheath-spine structure with transverse B-field in the central channel of the jet and longitudinal B-field at the edges is seen on epoch 2008.70. Right panel: Brightness temperature changes along the jet in 2155-152 at $15.4 \mathrm{GHz}$. Filled blue circles are the measured values. Open red circles are the predicted brightness temperatures from shock-in-jet model with adiabatic losses dominating the radio emission assuming predominantly transverse magnetic field orientation to the local jet direction. The errors are given at $3 \sigma$ level. 


\section{Conclusion}

The measured sizes and brightness temperatures of the VLBI components in parsec-scale jets in the radio galaxy $1128-047$ and the quasar 2155-047 are found to be consistent with emission from thin relativistic shocks dominated by adiabatic energy losses. The bright distinct features in these sources may indeed be a collection of relativistic shocks developing and expanding down the flow. Applying the shock-in-jet model to the VLBI observations we predict the predominantly transverse direction of the intrinsic magnetic field associated with the central channel of the jet in 1128-047. Discrepancy between the model and measured brightness temperatures for the bright jet component, located at $\sim 3.4$ mas from the core in 2155-152, can be explained either by reacceleration of the particles due to the interaction with the ambient medium or by changing the viewing angle that becomes nearly zero.

\section{References}

[1] S.A. Baum, C.P. O’Dea, G. Giovannini, et al., ApJ 483, 178 (1997).

[2] E.R. Craine, P.A. Strittmatter, S. Tapia, et al., ApL 17, 123 (1976).

[3] K.I. Kellermann, M.L. Lister, D.C. Homan, et al., ApJ 609, 539 (2004).

[4] Y.Y. Kovalev, K.I. Kellermann, M.L. Lister, et al., AJ 130, 2473 (2005).

[5] A.P. Marscher, Interpretation of Compact Jet Observations, in proceedings of Parsec-Scale Radio Jets, ed. J.A. Zensus \& T.J. Pearson (Cambridge: Cambridge Univ. Press 1990), 236.

[6] A.P. Marscher, Variability of the Non-thermal Emission in the Jets of Blazars, in proceedings of Blazar Continuum Variability, ASP Conference Series 110, ed. by H.R. Miller, J.R. Webb, and J.C. Noble, pp. 248-261 (1996).

[7] R. Laing, Brightness and Polarization Structure of Decelerating Relativistic Jets in proceedings of Energy Transport in Radio Galaxies and Quasars, ASP Conference Series 100, pp. 241-252 (1996).

[8] M.L. Lister et al., in prep (2009).

[9] A.P. Lobanov, E. Carrara, J.A. Zensus, Vistas in Astronomy 41, 253 (1997).

[10] A.P. Lobanov, T.P. Krichbaum, D.A. Graham, et al., $A \& A$ 364, 391 (2000).

[11] A.P. Lobanov, J.A. Zensus, Active Galactic Nuclei at the Crossroads of Astrophysics, in proceedings of Exploring the Cosmic Frontier: Astrophysical Instruments for the $21^{\text {st }}$ Century, ESO Astrophysical Symp. Series, ed. by A.P. Lobanov, J.A. Zensus, C. Cesarsky, P.J. Diamond, pp. 147-162 (2006).

[12] A.B. Pushkarev, D.C. Gabuzda, Yu.N. Vetukhnovskaya, and V.E. Yakimov, MNRAS 356, 859 (2005).

[13] E. Ros, J.A. Zensus, A.P. Lobanov, $A \& A$ 354, 55 (2000).

[14] T. Savolainen, Y.Y Kovalev, $A \& A$ 489, 33 (2008).

[15] M.C. Shepherd, Difmap: an Interactive Program for Synthesis Imaging, in proceedings of Astronomical Data Analysis Software and Systems VI, ASP Conference Series 125, ed. by Gareth Hunt and H.E. Payne, pp. 77-84 (1997).

[16] M.P. Véron-Cetty, P. Véron, $A \& A$ 455, 773 (2006).

[17] S.J. Wagner, A. Witzel, ARA\&A 33, 163 (1995).

[18] J.-H. Woo, M.C. Urry, ApJ 579, 530 (2002). 